\title{
Structures and Orientations of PHYSICS IN FRANCE
}

\section{A. Rousset, Paris}

(Scientific Adviser at the Délégation Générale à la Recherche Scientifique et Technique)

Physics in France is practised in very different laboratories: in the Universities, in the Centre National de Recherches Scientifique (C.N.R.S.), in the Commissariat à l'Energie Atomique (C.E.A.), in the schools of engineering. These bodies have also very different aims and the research varies correspondingly. University research is very diverse in all sectors; generally it consists of so-called "light physics" carried out essentially by teaching staff. The C.N.R.S. has a more concentrated objective and is a very efficient body in the field of fundamental research. Its researchers are employed full time, either in its own, or in associated university laboratories. The C.E.A. possesses extensive technical facilities and is well suited to the construction and the operation of large equipment, such as accelerators, nuclear reactors and big detectors. In the schools of engineering, physics research is mostly devoted to applications directly related to industrial needs.

French physicists can find in these bodies the necessary potential and the environment suited to their specific research aims. These bodies, which come under different ministries, need a coordinating agency, a role filled by the Délégation Générale à la Recherche Scientifique et Technique. The D.G.R.S.T. is answerable to the Prime Minister, through the office of the Secretary of State for Research, and so can play, without ambiguity, an interministerial role. Its responsibility ranges from the most fundamental research to applied research and industrial development. As a result, the essential links between all research and development levels can be ensured.

The essential mission of the D.G.R.S.T. is the coordination of research, taking into account social and economic demands raised by the scientific community, by industry, by social partners and by the ministries of: Education, Industry, Health, Agriculture, Transport, Cooperation, Foreign Affairs, etc. A complete analysis is made every five years in the Plans. Recently, the D.G.R.S.T. has had the task of preparing the VIIth Plan for
Research in concert with research bodies, ministries and trade unions. In the VIIth Plan are defined the priority orientations which will govern research policy. Also laid down are a new policy for scientific training and recruitment, a policy encouraging the development of jointly operated equipment and a policy covering the location of research. Unfortunately, annual budgets do not come up to the Plan forecasts and some objectives will probably not be met.

The D.G.R.S.T. has the duty to negociate with the Treasury each year, the total budget for French research and then distribute it among the different research bodies. In the present time of economic crisis, it is very difficult to obtain a physics budget on a par with that expected by the scientific community. The operational credits of laboratories and the equipment credits are not following the trends presaged in the VIIth Plan. Consequently, it is not easy under these conditions to support a dynamic policy in physics, all the more because this sector has only a very small overlap with the priority programmes. In particular, the big items of equipment are especially sensitive to a decrease in the budget.

A complementary task of the D.G.R.S.T. is the management of the incentive credits (about 300 MFF), generally orientated towards some specific, socio-economic objective in the long or medium term. The allocated credits are used particularly for applied research, but also for basic research which is just up-stream of application. For instance, a major effort is made to develop new components in electronics, opto-electronics, acousto-electronics, etc., but credits are also allocated to solid state laboratories, which are looking for new materials, methods of modifying the properties of materials, and understanding and controlling their characteristics. Another example is the research into high power lasers which will be necessary for controlled thermo-nuclear fusion by inertial confinement; credits are allocated to fundamental research laboratories which are trying new gases or new materials which might lead to lasers of higher power than those currently available.

Important decisions have been taken recently, which modify the evolution of physics in France. To start with, we shall consider university research which affects a very large number of teacher-researchers. This research is very diverse, it is excellent on the theoretical side but is inadequately supported on the experimental side, as funds are too limited and are uniformly distributed over too great a number of research workers. Through a re-organization of the management of university research, efficiency in this sector should improve in the near future. Over the past several years, the Universities have had no increase of staff. The average age of teacher-researchers has been going up and the mobility between Universities is very poor. The creation of some new posts where teachers can temporarily do full-time research for a limited period will introduce some degree of fluidity into a system which is presently frozen.

Research in the big bodies, like the C.N.R.S. or the C.E.A., has been seriously prejudiced by the quasi complete stopping of recruitment of young researchers between 1970 and 1975. This obliged teachers systematically to discourage any young student who was wishing to enter research. Since the "Conseil Restreint" of February 1975, the Government has set itself the objective of returning to a continuous recruitment rate of $3 \%$ per year for scientific staff. As a result, the age distribution will gradually improve and the number of young researchers will be brought into balance.

Another decision of the same Council, which encourages students to enter research, was the establishment of 1500 student fellowships per year, covering two years study for the preparation of a third cycle thesis. About 270 such fellowships will be allocated annually to young physicists.

Fundamental research is very often associated with big equipment. With the biggest instruments, matter can be explored with the highest preci- 
sion and with the greatest chance of making new discoveries. Equipment is becoming bigger and bigger, more and more complex, and consequently more and more expensive. The scientific community has to concentrate its means on a limited number of instruments, and these have to be chosen with great care. Latterly, French physicists have opted in favour of medium-sized facilities and this is illustrated by the examples given below.

In the nuclear physics field, an important decision was taken in 1975 , to construct at Caen, a national heavy ion accelerator, GANIL. This new machine will be able to provide heavy ion beams, from helium to uranium, with a good intensity $\left(10^{11}\right.$ to $10^{12}$ ion/s) and a good energy resolution $\left(10^{-3}\right.$ to $\left.10^{-4}\right)$. The energy per nucleon will extend from $100 \mathrm{MeV}$ for the relatively light ions to $10-20 \mathrm{MeV}$ for heavy ions. This energy, bigger than the Coulomb potential, will allow nuclear collisions at very high levels of collective excitation. New phenomena can be expected: multi-nucleon exchange, production of super-heavy elements, generation of shock waves in nuclear matter associated with the production of mesons, etc. The unique characteristics of GANIL will attract to Caen many French and other European physicists in the years 19811985.

Saturne, initially built for elementary particle physics, is currently being completely rebuilt, almost entirely for the use of nuclear physicists. A good definition of the incident proton energy and an accurate detection of the scattered protons will lead to a very precise analysis of the excitation levels of nuclei. This accelerator, at the border of two scientific fields, will be a unique instrument for analysing nuclear excitations at high energy.
Electron microscopy is a field which has been developed with much success at Toulouse. Several high voltage microscopes (from 1 to $3 \mathrm{MV}$ ) are used by physicists, but also by biologists, chemists, and metallurgists. A new high voltage scanning microscope is under construction. Only one other instrument similar to it will be operational in the next few years and that in the U.S.A. Unfortunately, budgetary restrictions mean that this important project cannot go ahead at the pace originally foreseen.

The construction of electron colliding beams by elementary particle physicists has brought a very important by-product: synchrotron radiation, which is used by other physicists as a source of far ultraviolet or X-rays. The existence of the Orsay machines ( $\mathrm{ACO}$ and $\mathrm{DCl}$ ) has justified the construction of big laboratories to use these very strong sources of photons (LURE).

Neutron beams to-day have wide application in crystallography, biology, physics and chemistry. The high flux reactor in Grenoble used jointly by British, German and French scientists is now the centre of a great deal of scientific activity. A new reactor (ORPHEE) with a lower level of performance, is currently under construction at Saclay for similar purposes. It will replace the aging EL3 reactor now more than twenty years old.

Research on controlled thermonuclear fusion is orientated at present towards two kinds of large scale experimentation: the magnetic confinement of high temperature plasma in the Tokamaks and inertial confinement by laser beam implosion. The Tokamak at Fontenay-aux-Roses has led to important advances in this field (25 ms confinement duration, $2000 \mathrm{eV}$ ion temperature); JET should provide the means to pursue this route and achieve thermonuclear conditions, if political divergences between members of the European Communities can be resolved. Research on fast fusion, by laser beam implosion, was carried out in France up to 1976 in military laboratories, but problems of collaboration have now led the civil laboratories to undertake their own research. As a result, they have a very important lag to catch up in order to reach the level of international competence of the Russians or Americans.

Elementary particle physics is a very active field in France. In the past it was the construction and the use of bubble chambers, thanks to the C.E.A., which were particularly well developed. Today the main effort continues to be directed towards experiments on the CERN accelerators, but emphasis should be laid on the construction of the $\mathrm{e}^{+} \mathrm{e}^{-}$colliding beam facility at Orsay with which recently the new particle $\omega^{\circ}$ was discovered, and the preparation of a major experiment at the future PETRA rings at Hamburg.

French physics has, it can be seen, three complementary aspects: the non-orientated research, principally located in the Universities; research round big instruments which requires from the scientific community a determined will to centralize its resources; orientated research devoted to social and economic objectives. When these three forms of research exist in the same laboratory, the situation is ideal. Indeed, applied research can then attain the highest quality, being stimulated by the proximity of both fundamental research and the powerful instruments. It is also desirable that conditions are created whereby researchers can pass easily from the fundamental field to the applied field and vice-versa. The D.G.R.S.T. must assure the necessary balance between these three complementary forms of research.

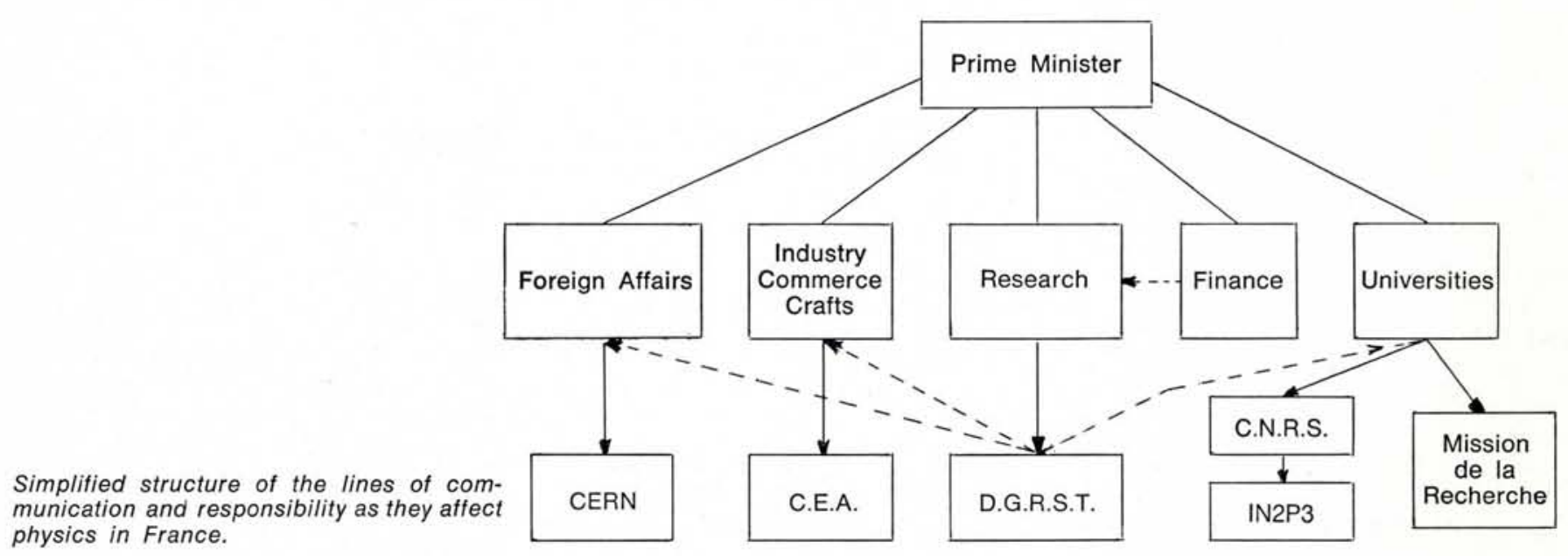

